A GENERAL DIAGRAMMATIC METHOD OF REPRESENTING PROPOSITIONS AND INFERENCE IN THE LOGIC OF CLASSES.

BY PROFESSOR L. M. HOSKINS.

(Read before the San Francisco Section of the American Mathematical Society, February 29, 1908.)

THE following method of representing class relations depends upon an analogy which is most simply explained in the case of propositions involving three class terms $x, y, z$.

Let the domain of any class $x$ and that of its negative $x^{\prime}$ be

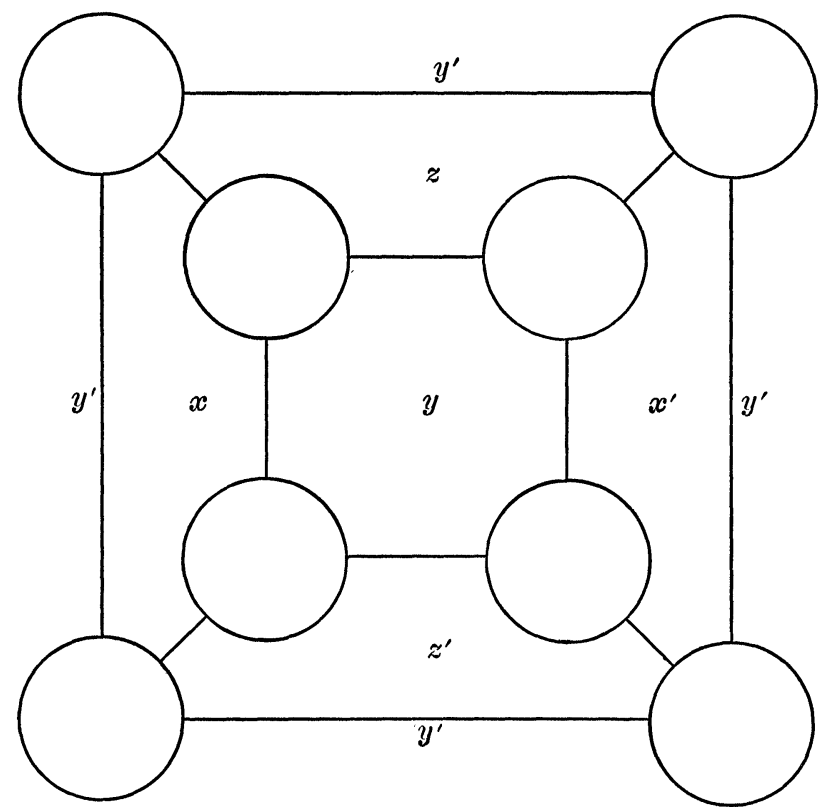

FIG. 1.

represented by two parallel faces of a cube, and let the other pairs of faces in like manner represent the domains of $y, y^{\prime}$ and $z, z^{\prime}$ respectively. Then the domain common to any two of the six classes $x, x^{\prime}, y, y^{\prime}, z, z^{\prime}$ may be represented by the edge in which the corresponding planes intersect, while the domain 
common to three of them will be represented by the vertex in which three planes intersect. This gives a symmetrical representation of the relations among the eight subclasses $x y z$, $x y z^{\prime}, \ldots$

For practical use in the solution of logical problems, a convenient diagram is obtained by drawing a plane projection of a cube and marking each face with its corresponding class symbol. At each vertex may be left a circular space which, in any given case, may receive a distinguishing mark in accordance with the import of a particular proposition. Such a diagram is represented in Fig. 1.

A symmetrical representation for the case of $n$ primary class terms would require a figure in $n$ dimensions. But a plane diagram for practical use may be made by repeating the diagram for the case of three terms. For example, the case of six primary terms may be represented by eight cubes, each placed at a vertex of a larger cube. Thus the projection shown in Fig. 1 might be drawn on a large scale, and a similar projection drawn in each of the circular spaces; each of these smaller figures representing the relations of three primary terms $u, v$, $w$ and their negatives.

For practical use a permanent diagram may be drawn upon a slate or blackboard, so that the marks used in particular problems may readily be erased.

\section{HEINRICH MASCHKE: HIS LIFE AND WORK.}

Heinrich Maschke was born at Breslau in Germany on October 24, 1853. He received his early education at the Maria-Magdalenen-Gymnasium of his native town, where his exceptional mathematical talent soon attracted the attention of his teachers. In 1872 he graduated from the gymnasium and entered the University of Heidelberg as a student of mathematics, the science which he had selected for his life study. After several semesters at Heidelberg, where he was initiated by Königsberger into the mysteries of infinitesimal calculus, and at Breslau, where he served his year as "Einjährig-Freiwilliger" in the Prussian army, he went for three years to to Berlin, attracted by the famous triad Weierstrass, Kummer, and Kronecker, of whom Kummer seems to have had the most lasting influence upon his mathematical development. 